of post-ERCP pancreatitis have yielded conflicting results, and prophylaxis might not be cost-effective for all patients. For the first time, a study has investigated the efficacy and costeffectiveness of routine prophylaxis for the prevention of post-ERCP pancreatitis.

In this retrospective study, 2,461 consecutive patients underwent ERCP either with $(n=1,149)$ or without $(n=1,312)$ gabexate infusion (begun 30-90 min before ERCP). Gabexate was chosen as the prophylactic agent, because it has shown efficacy in previous trials. Patients were characterized as standard risk $(n=1,477)$ or high risk $(n=984)$, on the basis of whether they had at least one known risk factor for post-ERCP pancreatitis.

Overall, post-ERCP pancreatitis was significantly less common in patients who received gabexate prophylaxis than in patients who did not $(2.2 \%$ versus $3.9 \%$, respectively, $P=0.019)$; however, when standard-risk and high-risk patients were analyzed separately, this difference was restricted to high-risk patients $(7.3 \%$ versus $3.8 \%$, respectively, $P=0.012$ ). Gabexate prophylaxis did not affect the severity of the pancreatitis.

Financial analysis also showed that gabexate prophylaxis was cost-effective only in highrisk patients - so routine prophylaxis is only recommended in these patients.

Original article Testoni PA et al. (2006) Frequency of postERCP pancreatitis in a single tertiary referral centre without and with routine prophylaxis with gabexate: a 6-year survey and cost-effectiveness analysis. Dig Liver Dis 38: 588-595

\section{Serum adiponectin as a marker for gallstones}

Cholesterol gallstones result from abnormal cholesterol metabolism (biliary cholesterol supersaturation), and pigment gallstones most commonly occur as a result of bacterial degradation and precipitation of biliary lipids after mechanical obstruction of the biliary tract. Altered adiponectin levels have been associated with metabolic abnormalities; low levels have been linked to cancers, obesity, and obesity-related disorders. Wang et al. have now investigated whether this adipokine has a role in cholelithiasis.

Their retrospective study included 58 patients with symptomatic cholesterol gallstones, 47 with symptomatic pigment gallstones, and
101 healthy controls. Blood samples were taken from controls during routine medical check-ups, and from patients with gallstones on the day of (but before) cholecystectomy. $A$ radioimmunoassay was used to measure serum adiponectin levels.

Compared with the control group, serum adiponectin levels were markedly lower in patients with cholesterol gallstones and markedly higher in patients with pigment gallstones. On multivariable analysis, female sex and low adiponectin levels were identified as predictors for cholesterol gallstone formation, whereas advanced age and high adiponectin levels were predictors for pigment gallstone formation.

The authors conclude that the formation of gallstones of different type is affected by the production of adiponectin, and suggest that the serum adiponectin level could be used as a biomarker for both cholesterol and pigment gallstones.

Original article Wang S-N et al. (2006) Serum adiponectin levels in cholesterol and pigment cholelithiasis. Br J Surg 93: 981-986

\section{HIV drug shows promise in HBV treatment}

Tenofovir disoproxil fumarate (tenofovir) is an antiviral agent currently approved for the treatment of HIV. A new study has now shown it to be surprisingly effective against HBV.

In a retrospective study, 20 patients with HBV, who had not responded to sequential therapy with lamivudine followed by adefovir dipivoxil, received tenofovir $300 \mathrm{mg}$ daily for up to 2 years. One patient's dose was reduced because of renal insufficiency. In the 19 patients who received the full dose, HBV DNA was suppressed to an undetectable level ( $<400$ copies $/ \mathrm{ml}$ ) after a median of 3.5 months of treatment. The median decrease in HBV DNA level at the end of each patient's observation period (median 1 year) was $-3.8 \pm 1.1 \log _{10}$ copies $/ \mathrm{ml}$. Of the 14 patients with elevated serum alanine aminotransferase levels before tenofovir treatment, 10 patients had normal levels by the end of the study. No tenofovir-related adverse effects were reported.

Although these data are impressive, the authors point out that they only apply to lamivudine-resistant patients who had persistently high levels of HBV replication 